\title{
Rare Repeated Recurrence of Parosteal Osteosarcoma Adjacent to a Prosthesis after 15 Years: A Case Report
}

\author{
Ahmad Rezaee Azandaryani' ${ }^{1}$, Leili Ebrahimi Farsangi2* ${ }^{*}$,abihollah Rahmanifar ${ }^{1}$, \\ Jalal Dastmalchi² \\ ${ }^{1}$ Hamadan University of Medical Science, Hamadan, Iran \\ ${ }^{2}$ Tehran University of Medical Science, Tehran, Iran \\ Email: lilieage@gmail.com
}

Received 31 March 2014; revised 30 April 2014; accepted 29 May 2014

Copyright (C) 2014 by authors and Scientific Research Publishing Inc.

This work is licensed under the Creative Commons Attribution International License (CC BY). http://creativecommons.org/licenses/by/4.0/

(c) (i) Open Access

\begin{abstract}
Late relapsing parosteal osteosarcoma has been reported very rarely; however, to the best of our knowledge, long-term repeated recurrence of this tumor has not been reported yet. Herein, we present a case of late relapsing low grade parosteal osteosarcoma after 15 years in popliteal cavity after advising tumor excision, removal of the organ involved, and the placement of prosthesis.
\end{abstract}

\section{Keywords}

\section{Repeated Recurrence, Parosteal Osteosarcoma}

\section{Introduction}

Parosteal osteosarcoma is a rare low-grade highly differentiated tumor which usually arises from periosseous tissues adjacent to the bone cortex that commonly appeared in the distal dorsal femur. Parosteal osteosarcomas make up $4 \%$ of all osteosarcomas and represent from $1.6 \%$ to $2.0 \%$ of all bone malignancies. It is frequently prevalent in second decade of life onwards and in spite of no sex predilection; some observations have suggested female preponderance [1]. Radiographic assessment is the initial diagnostic approach in the cases who suspected to this tumor. In line, magnetic resonance imaging (MRI) and computed tomography (CT) are both employed to diagnose and differentiate parosteal osteosarcomas especially for local staging as well as for evaluating intramedullary involvement [2]. The first choice treatment protocol for this tumor is wide resection with limb salvage, but other therapeutic approaches including chemotherapy and radiotherapy are generally not very effective for

\footnotetext{
*Corresponding author.
}

How to cite this paper: Azandaryani, A.R., Farsangi, L.E., Rahmanifar, Z. and Dastmalchi, J. (2014) Rare Repeated Recurrence of Parosteal Osteosarcoma Adjacent to a Prosthesis after 15 Years: A Case Report. Case Reports in Clinical Medicine, 3, 340-344. http://dx.doi.org/10.4236/crcm.2014.36075 
its treating. Meanwhile, despite high successfulness of surgical approach, it is contraindicated for those who suffered generalized metastasis and also may result in some significant complications such as impaired wound healing, local or systemic infections, hematoma, neurovascular injury, failure of prosthesis implantation, and recurrence.

Although this type of osteosarcoma has been reported to have a better prognosis than other variants, but it has a potential to turn into a high-grade poorly differentiated tumors as well as to rarely recur. Also, although it has a slow rate of development, it may eventually destroy the cortex and involves the medulla at later stages [3]. In addition, this malignancy has a high chance of recurrence if not removed properly. In this regard, late relapsing parosteal osteosarcoma has been reported very rarely; however, to the best of our knowledge, repeated recurrence of this tumor has not been reported yet.

Herein, we present a case of repeated recurrent parosteal osteosarcoma in the distal femur years after its initial excision and radiotherapy.

\section{Case Presentation}

A 35-year-old woman referred to the Ekbatan Hospital, Hamadan in 1995 with the history of lower limb trauma following car accident and reported severe pain in her left knee and also limitation of motion in the knee to a 60 degree. After a year and because of worsening symptoms, she assessed by X-Ray imaging techniques that a mass lesion as in popliteal cavity had subsequently been found in the left femur (Figure 1), which had been finally diagnosed as osteosarcoma by MRI and then confirmed by bone scan. The patient then referred to Sina hospital in Tehran for more assessment by bone biopsy that according to excisional biopsy, low grade parosteal osteosarcoma diagnosis was raised. Patient underwent chemotherapy for two times without observable treatment response, leading the decision to amputation or knee arthrodesis. The patient did not consent to do so and therefore all patients' documents and biopsy specimen were sent to Gustavo Rossi Institute in France for more evaluation that the recent diagnosis was also confirmed by this institute, but without recommending surgical amputation and with advised to tumor excision, removal of the organ involved, and the placement of prosthesis. The patient was thus programmed for this surgery in Royal National Orthopedic hospital (RNOH) in the United Kingdom that resulted in successful surgical artificial joint replacement of the knee. Patient remained asympto matic within 15 years later, however in 2010 and simultaneous to her second pregnancy, she reported a mass in

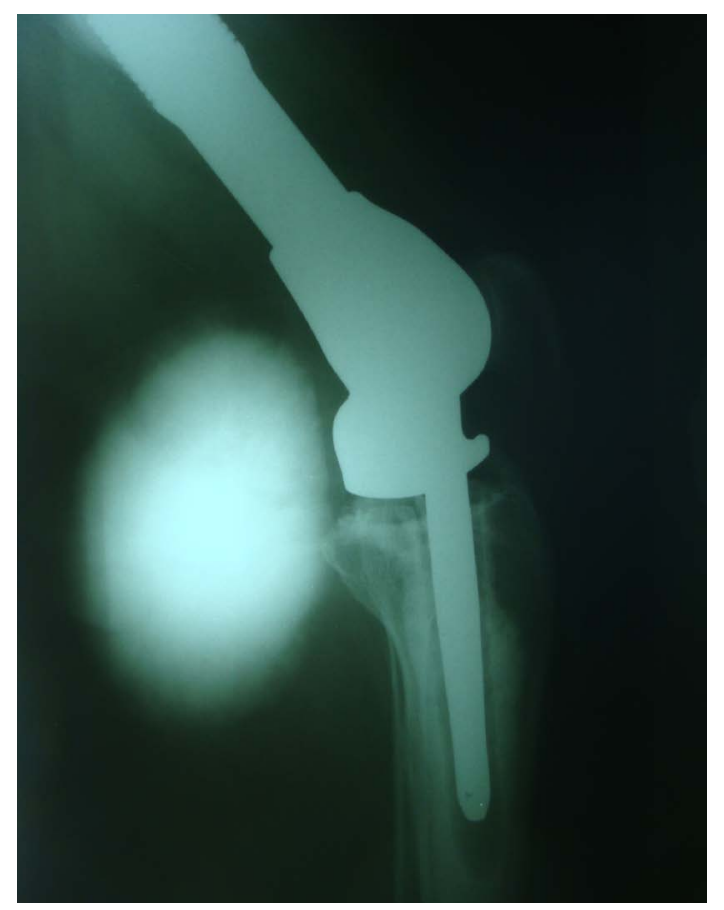

Figure 1. Lateral radiograph of left knee shows a large calcified mass at the popliteal cavity near the knee prosthesis. 
the posterior zone of the knee with the diameter $3 \times 3 \mathrm{~cm}$ with gradually progressive limitation in knee flexion. Within the first trimester, she underwent needle biopsy leading diagnosis of parosteal osteosarcoma. Based on the doctor recommendation, no attempt was made in surgery to terminate pregnancy. During pregnancy, the mass began to growing and enlargement and at the end of pregnancy (thirty-six week of pregnancy); tumor growing was appeared to be rapid and more progressive. X-Ray assessment was made after delivery indicating a mass with the diameter $20 \times 20 \mathrm{~cm}$ (Figure 1). CT angiography showed a large vascular mass with high probability of parosteal osteosarcoma (Figures 2(a)-(c)). Patient underwent surgical excision of the mass successfully without any involvement of the surrounding vessels and nerves (Figure 3). Excisional biopsy again confirmed low grade parosteal osteosarcoma diagnosis indicating long-term recurrence after joint replacement. Three months later and using diagnostic imaging, two suspicious calcifications were revealed in the posterior knee area (Figure 4). The patient was finally scheduled for radiation therapy for 30 sessions that is now continuing.

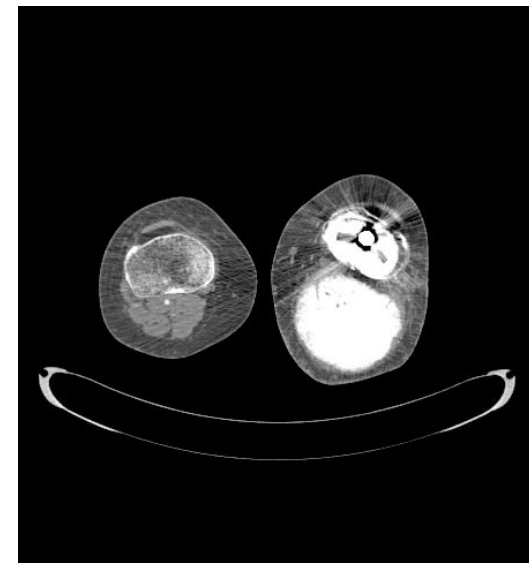

(a)

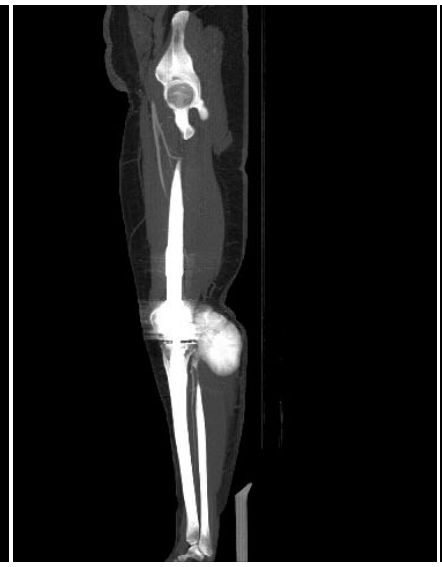

(b)

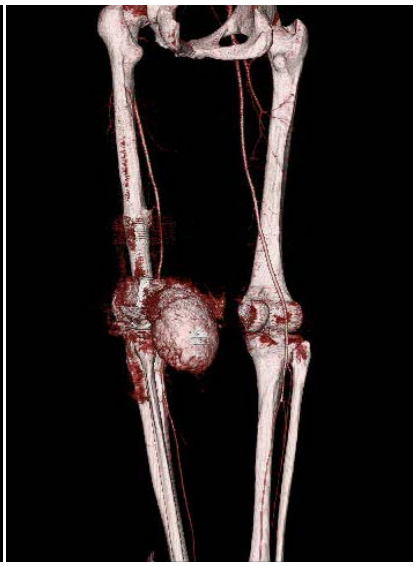

(c)

Figure 2. Axial (a), sagittal MPR (b) and volume rendering (c) contrast enhanced CT images of the knee shows a large calcified mass in popliteal cavity near the prosthesis.

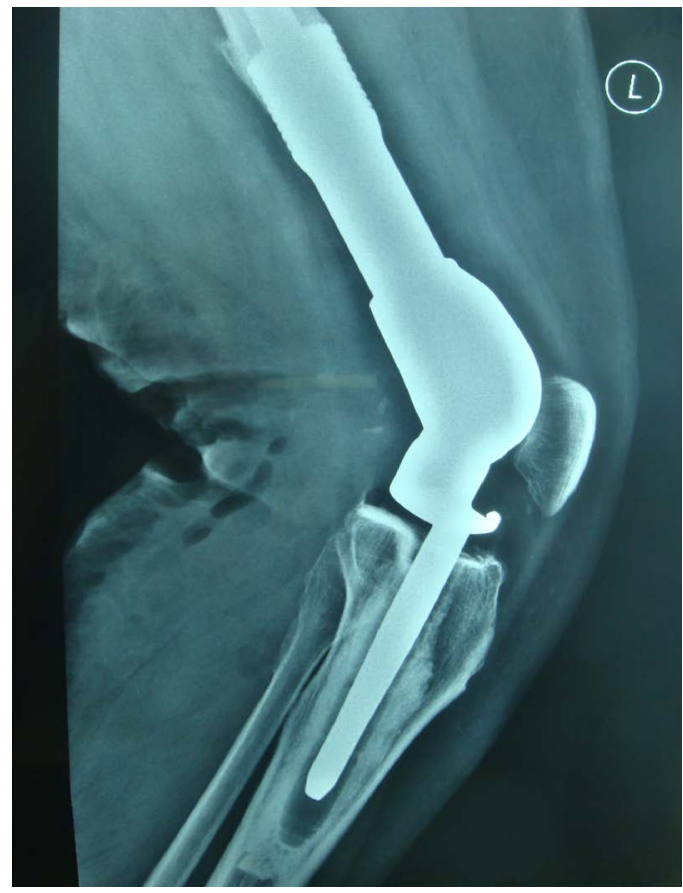

Figure 3. Lateral radiograph of left knee after complete resection of the mass. 


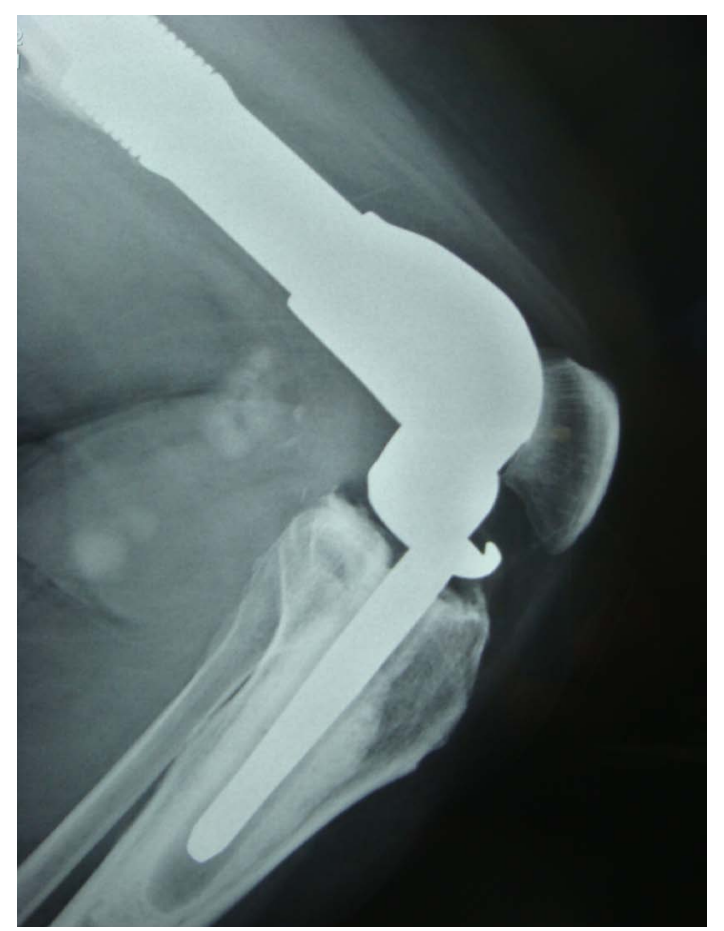

Figure 4. Same view 3 months later shows two suspicious foci of calcifications were revealed in the posterios knee area.

\section{Discussion}

Because of low-grade nature of parosteal osteosarcoma, better prognosis of this tumor compared with other types of osteosarcomas is predictable. For all low-grade lesions, wide resection of affected area seems to be completely curative. However, in high-grade areas, the prognosis may approach that of conventional high-grade osteosarcomas, and the patient requires radiochemotherapy in addition to surgical excision. In surgical approach, mega-prosthesis implantation is recommended, but rare late relapse or even late repeated recurrence of parosteal osteosarcoma may occur. In a study by Lau et al. [4], a case of parosteal osteosarcoma was reported adjacent to prosthesis after 20 years that was successfully treated surgically and regained muscle strength and range of motion of the knee 6 months after surgery. After three-year follow-up, she remained asymptomatic and had excellent knee function. Okada et al. [3] also reported that the longest time from definitive surgery to local recurrence was 15 years. Furthermore, Temple et al. [5] reported a mean of local recurrence at 7.6 years from surgery. However, none of the studies showed repeated recurrences within follow-up time. In our case, recurrences were occurred after 1, 8, and 15 years after initial assessment of lesion. It seems that the times to recurrence may be dependant to therapeutic approach including the adequacy of the surgery, diagnostic accuracy and correct differential diagnosis, as well as to the length of follow-up. The presented study was treated with only surgical approach that also remained challenging in each schedule because of no detected metastasis, slow growing feature, and diagnosing local lesions as calcification or bleeding. However, final approach with radiotherapy following surgical resection seemed to be useful and effective. In total, long-term vigilance and follow-up is needed to assess repeated recurrences and response to treatment.

\section{References}

[1] Lorentzon, R., Larsson, S.E. and Boquist, L. (1980) Parosteal (Juxtracortical) Osteosarcoma. A Clinical and Histopathological Study of 11 Cases and Review of the Literature. The Journal of Bone \& Joint Surgery, 62, 86-92.

[2] Jelinek, J.S., Murphey, M.D. and Kransdorf, M.J. (1996) Parosteal Osteosarcoma: Value of MR Imaging and CT in the Prediction of Histologic Grade. Radiology, 201, 837-842.

[3] Okada, K., Frassica, F.J., Sim, F.H., Beabout, J.W., Bond, J.R. and Unni, K.K. (1994) Parosteal Osteosarcoma. A Clinicopathological Study. The Journal of Bone \& Joint Surgery, 76, 366-378. 
A. R. Azandaryani et al.

[4] Lau, T., Wong, J., Yip, D. and Chien, E. (2004) Local Recurrence of Parosteal Osteosarcoma Adjacent to Prosthesis after 20 Years: A Case Report. Journal of Orthopaedic Surgery, 12, 263-266.

[5] Temple, H.T., Scully, S.P., O’Keefe, R.J., Katapurum, S. and Mankin, H.J. (2000) Clinical Outcome of 38 Patients with Juxtacortical Osteosarcoma. Clinical Orthopaedics, 373, 208-217.

http://dx.doi.org/10.1097/00003086-200004000-00025 